PROCEEDINGS OF THE

AMERICAN MATHEMATICAL SOCIETY

Volume 137, Number 11, November 2009, Pages 3695-3702

S 0002-9939(09)09987-0

Article electronically published on June 12, 2009

\title{
SYMMETRY OF INTEGRAL EQUATIONS ON BOUNDED DOMAINS
}

\author{
DONGSHENG LI, GERHARD STRÖHMER, AND LIHE WANG
}

(Communicated by Matthew J. Gursky)

\begin{abstract}
In this paper, we will investigate the symmetry of both domains and solutions of integral equations on bounded domains via the method of moving planes.
\end{abstract}

\section{IntRoduction AND the MAIN RESUlt}

Let $n$ be a positive integer and $\Omega \subset R^{n}$ be a bounded domain. We will consider the following integral equations:

$$
\left\{\begin{array}{l}
u(x)=A \int_{\Omega} \frac{|u|^{p}(y)}{|x-y|^{n-\alpha}} d y+B, \\
u=\beta \text { on } \partial \Omega,
\end{array}\right.
$$

where $p, \alpha, \beta, A$ and $B$ are constants satisfying

$$
p, A>0, \quad \beta, B \geq 0 \text { and } 1<\alpha<n .
$$

Regarding the right-hand side of (1.1) as an integral operator of $u$, if $p \leq 1$, then it is easy to see that the operator is bounded from $L^{1}(\Omega)$ into $L^{1}(\Omega)$. Actually,

$$
\begin{gathered}
\int_{\Omega}\left|A \int_{\Omega} \frac{|u|^{p}(y)}{|x-y|^{n-\alpha}} d y+B\right| d x \leq \frac{A d^{\alpha} n \omega_{n}}{\alpha} \int_{\Omega}|u|^{p}(y) d y+B|\Omega| \\
\leq \frac{A d^{\alpha} n \omega_{n}}{\alpha}|\Omega|^{1-p} \int_{\Omega}|u|(y) d y+B|\Omega|,
\end{gathered}
$$

where $d$ denotes the diameter of $\Omega$ and $\omega_{n}$ the volume of the unit ball in $R^{n}$. If $p>1$, from the Hardy-Littlewood-Sobolev inequality, it follows that the operator is bounded from $L^{q}(\Omega)$ into $L^{q}(\Omega)$ for any $q>\max \left\{\frac{n}{n-\alpha}, \frac{n}{\alpha}(p-1)\right\}$. Actually, let $\frac{1}{q}+\frac{\alpha}{n}=\frac{1}{r}$ and then $1<r$ and $p r<q$, which are implied by $q>\frac{n}{n-\alpha}$ and $q>\frac{n}{\alpha}(p-1)$, respectively. It follows from the Hardy-Littlewood-Sobolev inequality

Received by the editors August 13, 2008.

2000 Mathematics Subject Classification. Primary 45K05, 45P05; Secondary 35J67.

Key words and phrases. Symmetry, integral equations, moving planes.

The first author was supported in part by NSF of China Grant \#10771166.

The second author was supported in part by PCSR Grant \#2 PO3A 002223.

The third author was supported in part by NSF Grant \# DMS-0701392. 
and the Hölder inequality that

$$
\begin{gathered}
\int_{\Omega}\left|A \int_{\Omega} \frac{|u|^{p}(y)}{|x-y|^{n-\alpha}} d y+B\right|^{q} d x \leq 2^{q-1}\left\{A^{q}\left(\int_{\Omega}|u|^{p r}(y) d y\right)^{\frac{q}{r}}+B^{q}|\Omega|\right\} \\
\leq 2^{q-1}\left\{A^{q}|\Omega|^{1-\frac{p r}{q}}\left(\int_{\Omega}|u|^{q}(y) d y\right)^{p}+B^{q}|\Omega|\right\} .
\end{gathered}
$$

Therefore, it is natural to assume that

$$
u \in L^{q}(\Omega), \text { where }\left\{\begin{array}{l}
q=1 \text { if } p \leq 1 \\
q>\max \left\{\frac{n}{n-\alpha}, \frac{n}{\alpha}(p-1)\right\} \text { if } p>1 .
\end{array}\right.
$$

Equation (1.1) arises from minimizing some energy functionals. For example, as $\alpha=2$, it is the Euler-Lagrange equation for the energy functional of a barotropic self-gravitating gas contained in the domain $\Omega$, where $|u|^{\frac{1}{p}}$ is the density of the gas and $\beta$ is the pressure outside $\Omega$ (cf. 4 4 ). In this case, it is also equivalent to the stationary compressible Navier-Stokes equations of the gas (cf. [5]).

Our purpose in this paper is to apply the method of moving planes to investigate the symmetry not only of solutions $u$ but also of domains $\Omega$ of (1.1). The method of moving planes has been extensively used to study the symmetry of solutions of differential equations. Here we only mention the original papers [2] and [3]. Some other important results in this direction are listed in the references of [1]. To the best of the authors' knowledge, [1 is the first approach to study the symmetry of integral equations via the method of moving planes, where Chen, $\mathrm{Li}$ and $\mathrm{Ou}$ obtained the symmetry of solutions $u$ of (1.1) as $\Omega=R^{n}$ and $p=\frac{n+\alpha}{n-\alpha}$ and also classified the solutions. As for integral equations on bounded domains, we will obtain the symmetry of both solutions and domains.

Our main result is the following:

Theorem 1.1. Suppose $\Omega \subset R^{n}$ is a $C^{1}$ bounded domain and (1.1), (1.2) and (1.3) hold. If $u>0$ in $\Omega$, then $\Omega$ is a ball and $u$ is radially symmetric and monotone decreasing about the center of the ball.

Remark 1.2. (i) From the proof of Theorem 1.1, we will see that Theorem 1.1 still holds if in (1.1) $|u|^{p}$ is replaced by $|f(u)|$ provided $f$ is positive and increasing on $[0, \infty)$ and there exist positive constants $p$ and $C$ such that

$$
\left\{\begin{array}{l}
\text { (i) } f(u) \leq C\left(1+u^{p}\right) \text { for any } u \geq 0, \text { and } \\
\text { (ii) } f(u)-f(v) \leq C\left(u^{p}-v^{p}\right) \text { for any } 0 \leq v \leq u .
\end{array}\right.
$$

(ii) It is clear that (1.1) implies the following differential equations:

$$
\left\{\begin{array}{l}
(-\Delta)^{\frac{\alpha}{2}} u=A u^{p} \text { in } \Omega, \\
u=\beta \text { on } \partial \Omega ;
\end{array}\right.
$$

however the inverse is not true since (1.4) implies indeed

$$
u(x)=A \int_{\Omega} \frac{|u|^{p}(y)}{|x-y|^{n-\alpha}} d y+\tilde{B}(x),
$$

where $\tilde{B}(x)$ usually cannot be a constant. To regain (1.1) from (1.4), we need some extra conditions; that is, the system should be overdetermined. Serrin [3] 
studied these kinds of problems and proved that $\Omega$ must be a ball. From this point of view, we see that integral equations usually give us more information than differential equations. Ströhmer [5] solved (1.1) with $u$ and $\Omega$ as unknowns under the constraint that $\int_{\Omega}|u|^{\frac{1}{p}}$ equals a given positive number.

(iii) In (1.2), we assume that $1<\alpha<n$ instead of $0<\alpha<n$, a more general assumption for $\alpha$ (cf. [1), since $1<\alpha$ implies $u \in C^{1}(\bar{\Omega})$ (cf. Lemma 2.2), which is needed in our proof of Theorem 1.1.

\section{A preliminary RESUlt}

In this section, we show that our assumptions in Theorem 1.1 imply $u \in C^{1}(\bar{\Omega})$. We first prove a simple lemma.

Lemma 2.1. Suppose $w \in L^{r}(\Omega)$ with $1 \leq r<\infty$ and

$$
v(x)=A \int_{\Omega} \frac{|w|(y)}{|x-y|^{n-\alpha}} d y+B .
$$

Then $v \in W^{1, s}(\Omega)$, where $\frac{1}{s}+\frac{\alpha-1}{n}=\frac{1}{r}$ if $1 \leq r<\frac{n}{\alpha-1}$, and $s$ can be any real number greater than 1 if $r \geq \frac{n}{\alpha-1}$.

Proof. Without loss of generality, we assume that $w \in C_{0}^{\infty}(\Omega)$. Then we have

It follows that

$$
\partial_{x_{i}} v(x)=-A(n-\alpha) \int_{\Omega} \frac{|w|(y)\left(x_{i}-y_{i}\right)}{|x-y|^{n-\alpha+2}} d y .
$$

$$
\left|\partial_{x_{i}} v(x)\right| \leq A(n-\alpha) \int_{\Omega} \frac{|w|(y)}{|x-y|^{n-\alpha+1}} d y .
$$

If $1 \leq r<\frac{n}{\alpha-1}$, from the Hardy-Littlewood-Sobolev inequality, we have

$$
\|D v\|_{L^{s}(\Omega)} \leq A(n-\alpha) C\|w\|_{L^{r}(\Omega)},
$$

where $\frac{1}{s}+\frac{\alpha-1}{n}=\frac{1}{r}$ and $C>0$ is a constant depending only on $n, s$ and $\alpha$. If $r \geq \frac{n}{\alpha-1}$, for any $s>\frac{n}{n-\alpha+1}$, let $\frac{1}{s}+\frac{\alpha-1}{n}=\frac{1}{r^{\prime}}$ and then $1<r^{\prime}<\frac{n}{\alpha-1} \leq r$. From the Hardy-Littlewood-Sobolev inequality and the Hölder inequality,

$$
\|D v\|_{L^{s}(\Omega)} \leq A(n-\alpha) C|| w\left\|_{L^{r^{\prime}}(\Omega)} \leq A(n-\alpha) C|\Omega|^{\frac{1}{r^{\prime}}-\frac{1}{r}}\right\| w \|_{L^{r}(\Omega)},
$$

where $C>0$ is a constant depending only on $n, s$ and $\alpha$.

In view of (2.1) (recall that $d$ is the diameter of $\Omega$ and $\omega_{n}$ is the volume of the unit ball in $R^{n}$ ),

$$
\|v\|_{L^{1}(\Omega)} \leq \frac{A d^{\alpha} n \omega_{n}}{\alpha}\|w\|_{L^{1}(\Omega)}+B|\Omega| \leq \frac{A d^{\alpha} n \omega_{n}}{\alpha}|\Omega|^{1-\frac{1}{r}}\|w\|_{L^{r}(\Omega)}+B|\Omega| .
$$

Combining this with (2.2) and (2.3), using Sobolev imbedding (note that $\Omega$ is bounded), we obtain the conclusion clearly.

Lemma 2.2. $u \in C^{1}(\bar{\Omega})$.

Proof. We first prove $u \in C(\bar{\Omega})$, which is sufficient to show $u \in L^{q^{\prime}}(\Omega)$ for some $q^{\prime}$ satisfying $\frac{q^{\prime}}{p} \geq \frac{n}{\alpha-1}$. Actually, since $u^{p} \in L^{\frac{q^{\prime}}{p}}(\Omega)$, from (1.1) and Lemma 2.1 with $s=n+1$, we obtain $u \in W^{1, n+1}(\Omega) \subset C(\bar{\Omega})$. Therefore we only need to consider the case that $\frac{q}{p}<\frac{n}{\alpha-1}$. Let $\frac{1}{s}+\frac{\alpha-1}{n}=\frac{p}{q}$ and from Lemma 2.1, we deduce $u \in W^{1, s}(\Omega)$. Using Sobolev embedding and the standard 'bootstrap' method, we will arrive at $u \in L^{q^{\prime}}(\Omega)$, where $q^{\prime}$ satisfies $\frac{q^{\prime}}{p} \geq \frac{n}{\alpha-1}$. 
Now we turn to show $u \in C^{1}(\bar{\Omega})$. Fix $\eta \in C_{0}^{\infty}(R)$ satisfying $0 \leq \eta \leq 1$ and $\eta(t)=0$ as $|t| \leq 1$, and $\eta(t)=1$ as $|t| \geq 2$. Define for any $\epsilon>0$,

$$
u_{\epsilon}=A \int_{\Omega} \frac{\eta_{\epsilon}|u|^{p}(y)}{|x-y|^{n-\alpha}} d y+B, \quad \eta_{\epsilon}=\eta(|x-y| / \epsilon) .
$$

From $u \in C(\bar{\Omega})$ and $\alpha>1$, we deduce that $\int_{\Omega} \frac{|u|^{p}(y)\left(x_{i}-y_{i}\right)}{|x-y|^{n-\alpha+2}} d y$ is integrable and

$$
u_{\epsilon} \rightarrow u, \quad \partial_{x_{i}} u_{\epsilon} \rightarrow-A(n-\alpha) \int_{\Omega} \frac{|u|^{p}(y)\left(x_{i}-y_{i}\right)}{|x-y|^{n-\alpha+2}} d y
$$

uniformly in $\Omega$ as $\epsilon \rightarrow 0$. Hence $u \in C^{1}(\bar{\Omega})$ and $\partial_{x_{i}} u=-A(n-\alpha) \int_{\Omega} \frac{\mid u p^{p}(y)\left(x_{i}-y_{i}\right)}{|x-y|^{n-\alpha+2}} d y$.

\section{Proof of Theorem 1.1}

We use the method of moving planes to prove Theorem 1.1. Choose any direction and rotate the coordinate system if it is necessary such that the $x_{1}$-axis is parallel to it. For any $\lambda \in R$, set

$$
T_{\lambda}=\left\{\left(x_{1}, x_{2}, \ldots, x_{n}\right): x_{1}=\lambda\right\}
$$

to be the hyperplane vertical to the $x_{1}$-axis. For $\lambda$ being sufficiently large, since $\Omega$ is bounded, we have that the hyperplane $T_{\lambda}$ is disjoint from $\bar{\Omega}$. Let the hyperplane $T_{\lambda}$ move continuously toward $\Omega$, i.e. decrease $\lambda$ continuously, until it begins to intersect $\bar{\Omega}$. Let $\lambda_{0}$ be such a $\lambda$; that is,

$$
\lambda_{0}=\max \left\{\lambda: T_{\lambda} \cap \bar{\Omega} \neq \emptyset\right\} .
$$

From that moment on, at every stage $T_{\lambda}$ will cut off from $\Omega$ an open cap:

$$
\Sigma_{\lambda}=\left\{\left(x_{1}, x_{2}, \ldots, x_{n}\right) \in \Omega: x_{1}>\lambda\right\} .
$$

For any $x=\left(x_{1}, x_{2}, \ldots, x_{n}\right)$, set $x^{\lambda}=\left(2 \lambda-x_{1}, x_{2}, \ldots, x_{n}\right)$ to be the reflection of $x$ about the hyperplane $T_{\lambda}$. Set

$$
\Sigma_{\lambda}^{\prime}=\left\{x^{\lambda} \in \Sigma_{\lambda}\right\}
$$

to be the reflection of $\Sigma_{\lambda}$ about $T_{\lambda}$. Since $\Omega$ is $C^{1}$, at the beginning, $\Sigma_{\lambda}^{\prime}$ will remain in $\Omega$, at least until one of the following occurs:

$$
\left\{\begin{array}{l}
(i) \Sigma_{\lambda}^{\prime} \text { is internally tangent to } \partial \Omega \text { at some point } \tilde{z} \text { not on } T_{\lambda} ; \\
\text { (ii) } T_{\lambda} \text { is orthogonal to the boundary of } \Omega \text { at some point } \hat{z} \text {. }
\end{array}\right.
$$

Denote by $\lambda_{1}$ the first value of $\lambda$ such that $T_{\lambda}$ reaches one of the above positions.

In what follows, we will show that

$$
\left\{\begin{array}{l}
\text { (i) } u\left(x^{\lambda}\right)>u(x) \text { for any } \lambda_{1}<\lambda<\lambda_{0} \text { and } x \in \Sigma_{\lambda} ; \\
\text { (ii) } \Sigma_{\lambda_{1}} \cup\left(\Omega \cap T_{\lambda_{1}}\right) \cup \Sigma_{\lambda_{1}}^{\prime}=\Omega ; \\
\text { (iii) } u\left(x^{\lambda_{1}}\right)=u(x) \text { for any } x \in \Sigma_{\lambda_{1}} .
\end{array}\right.
$$

Since $x_{1}$ can be any direction, Theorem 1.1 is a direct consequence of (3.2). 
For any $\lambda_{1}<\lambda<\lambda_{0}$ and $x, y \in \Sigma_{\lambda}$, since $\left|x-y^{\lambda}\right|=\left|x^{\lambda}-y\right|$ and $\left|x^{\lambda}-y^{\lambda}\right|=|x-y|$, from (1.1), we rewrite $u$ at $x$ and at $x^{\lambda}$ as (3.3)

$$
\left\{\begin{array}{l}
u(x)=A \int_{\Sigma_{\lambda}}\left(\frac{u^{p}(y)}{|x-y|^{n-\alpha}}+\frac{u^{p}\left(y^{\lambda}\right)}{\left|x^{\lambda}-y\right|^{n-\alpha}}\right) d y+A \int_{\Omega_{\lambda}} \frac{u^{p}(y)}{|x-y|^{n-\alpha}} d y+C \text { and } \\
u\left(x^{\lambda}\right)=A \int_{\Sigma_{\lambda}}\left(\frac{u^{p}(y)}{\left|x^{\lambda}-y\right|^{n-\alpha}}+\frac{u^{p}\left(y^{\lambda}\right)}{|x-y|^{n-\alpha}}\right) d y+A \int_{\Omega_{\lambda}} \frac{u^{p}(y)}{\left|x^{\lambda}-y\right|^{n-\alpha}} d y+C
\end{array}\right.
$$

respectively, where

$$
\Omega_{\lambda}=\Omega \backslash\left(\overline{\Sigma_{\lambda} \cup \Sigma_{\lambda}^{\prime}}\right) .
$$

We establish (3.2) by the following five lemmas.

Lemma 3.1. Suppose $\lambda_{1}<\lambda<\lambda_{0}$. If $\lambda_{0}-\lambda$ is small enough, then $u(x) \leq u\left(x^{\lambda}\right)$ for any $x \in \Sigma_{\lambda}$.

Proof. From (3.3) and $\left|x^{\lambda}-y\right|<|x-y|$ for any $x \in \Sigma_{\lambda}$ and $y \in \Omega_{\lambda}$, we deduce

$$
u(x)-u\left(x^{\lambda}\right)<A \int_{\Sigma_{\lambda}}\left(u^{p}(y)-u^{p}\left(y^{\lambda}\right)\right)\left(\frac{1}{|x-y|^{n-\alpha}}-\frac{1}{\left|x^{\lambda}-y\right|^{n-\alpha}}\right) d y .
$$

Set

$$
\Sigma_{\lambda}^{-}=\left\{x \in \Sigma_{\lambda}: u(x)>u\left(x^{\lambda}\right)\right\} .
$$

From $|x-y|<\left|x^{\lambda}-y\right|$ for any $x \in \Sigma_{\lambda}$ and $y \in \Sigma_{\lambda}$, it follows that

$$
u(x)-u\left(x^{\lambda}\right)<A \int_{\Sigma_{\lambda}^{-}}\left(u^{p}(y)-u^{p}\left(y^{\lambda}\right)\right) \frac{1}{|x-y|^{n-\alpha}} d y .
$$

We claim that if $\lambda_{0}-\lambda$ is small enough, then (3.4) implies

$$
\left|\Sigma_{\lambda}^{-}\right|=0 \text {. }
$$

Hence $\Sigma_{\lambda}^{-}=\emptyset$; that is, $u(x) \leq u\left(x^{\lambda}\right)$ for any $x \in \Sigma_{\lambda}$.

Actually, if $p \leq 1$, from (3.4), it follows that

$$
u(x)-u\left(x^{\lambda}\right) \leq \int_{\Sigma_{\lambda}^{-}}\left(u(y)-u\left(y^{\lambda}\right)\right)^{p} \frac{1}{|x-y|^{n-\alpha}} d y .
$$

Taking the $L^{1}$ norm on $\Sigma_{\lambda}^{-}$on both sides, we have

$$
\left\|u(x)-u\left(x^{\lambda}\right)\right\|_{L^{1}\left(\Sigma_{\lambda}^{-}\right)} \leq A\left\|\left(u(x)-u\left(x^{\lambda}\right)\right)^{p}\right\|_{L^{1}\left(\Sigma_{\lambda}^{-}\right)} \sup _{y \in \Sigma_{\lambda}^{-}} \int_{\Sigma_{\lambda}^{-}} \frac{1}{|x-y|^{n-\alpha}} d x .
$$

For any $y \in \Sigma_{\lambda}^{-}$, by rearrangement,

$$
\int_{\Sigma_{\lambda}^{-}} \frac{1}{|x-y|^{n-\alpha}} d x \leq n \omega_{n} \int_{0}^{d^{*}} r^{\alpha-1} d r=\frac{n \omega_{n}\left(d^{*}\right)^{\alpha}}{\alpha}
$$

where $\omega_{n}\left(d^{*}\right)^{n}=\left|\Sigma_{\lambda}^{-}\right|$. Hence

$$
\left\|u(x)-u\left(x^{\lambda}\right)\right\|_{L^{1}\left(\Sigma_{\lambda}^{-}\right)} \leq \frac{A n \omega_{n}^{1-\frac{\alpha}{n}}}{\alpha}\left|\Sigma_{\lambda}^{-}\right|^{1-p+\frac{\alpha}{n}}|| u(x)-u\left(x^{\lambda}\right) \|_{L^{1}\left(\Sigma_{\lambda}^{-}\right)} .
$$

Let $\lambda_{0}-\lambda$ be small enough such that $\left|\Sigma_{\lambda}^{-}\right|^{1-p+\frac{\alpha}{n}} \leq\left|\Sigma_{\lambda}\right|^{1-p+\frac{\alpha}{n}} \leq \frac{\alpha}{2 A n \omega_{n}^{1-\frac{\alpha}{n}}}$. Then

$$
\left\|u(x)-u\left(x^{\lambda}\right)\right\|_{L^{1}\left(\Sigma_{\lambda}^{-}\right)} \leq \frac{1}{2}\left\|u(x)-u\left(x^{\lambda}\right)\right\|_{L^{1}\left(\Sigma_{\lambda}^{-}\right)},
$$

which implies (3.5). 
If $p>1$, from (3.4), we have

$$
u(x)-u\left(x^{\lambda}\right)<p A \int_{\Sigma_{\lambda}^{-}} u^{p-1}(y)\left(u(y)-u\left(y^{\lambda}\right)\right) \frac{1}{|x-y|^{n-\alpha}} d y .
$$

From the Hardy-Littlewood-Sobolev inequality, it follows that

$$
\left\|u(x)-u\left(x^{\lambda}\right)\right\|_{L^{r}\left(\Sigma_{\lambda}^{-}\right)} \leq C\left\|u^{\frac{n}{\alpha}(p-1)}\right\|\left\|_{L^{\frac{n}{\alpha}(p-1)}\left(\Sigma_{\lambda}^{-}\right)}^{p-1}\right\| u(x)-u\left(x^{\lambda}\right) \|_{L^{r}\left(\Sigma_{\lambda}^{-}\right)}
$$

for any $\frac{n}{n-\alpha}<r \leq q$, where $C>0$ is a constant depending only on $r, n$ and $\alpha$. Let $\lambda_{0}-\lambda$ be small enough such that

$$
C|| u^{\frac{n}{\alpha}(p-1)}||_{L^{\frac{n}{\alpha}(p-1)}\left(\Sigma_{\lambda}^{-}\right)}^{p-1} \leq \frac{1}{2}
$$

and then (3.5) follows from (3.6).

Lemma 3.2. Suppose $\lambda_{1}<\lambda<\lambda_{0}$ and $u(x) \leq u\left(x^{\lambda}\right)$ for any $x \in \Sigma_{\lambda}$. Then $u(x)<u\left(x^{\lambda}\right)$ for any $x \in \Sigma_{\lambda}$.

Proof. From (3.3), it follows that

$$
\begin{aligned}
u\left(x^{\lambda}\right)-u(x) & =A \int_{\Sigma_{\lambda}}\left(u^{p}\left(y^{\lambda}\right)-u^{p}(y)\right)\left(\frac{1}{|x-y|^{n-\alpha}}-\frac{1}{\left|x^{\lambda}-y\right|^{n-\alpha}}\right) d y \\
& +A \int_{\Omega_{\lambda}} u^{p}(y)\left(\frac{1}{\left|x^{\lambda}-y\right|^{n-\alpha}}-\frac{1}{|x-y|^{n-\alpha}}\right) d y .
\end{aligned}
$$

Since $u(x) \leq u\left(x^{\lambda}\right)$ for any $x \in \Sigma_{\lambda}$ and $\Omega_{\lambda} \neq \emptyset$ (implied by $\lambda_{1}<\lambda<\lambda_{0}$ ), we have

$$
u\left(x^{\lambda}\right)-u(x) \geq A \int_{\Omega_{\lambda}} u^{p}(y)\left(\frac{1}{\left|x^{\lambda}-y\right|^{n-\alpha}}-\frac{1}{|x-y|^{n-\alpha}}\right) d y>0 .
$$

Lemma 3.3. Suppose $\lambda_{1}<\lambda<\lambda_{0}$ and $u(x)<u\left(x^{\lambda}\right)$ for any $x \in \Sigma_{\lambda}$. Then there exists $\epsilon>0$ such that $u(x) \leq u\left(x^{\tilde{\lambda}}\right)$ for any $x \in \Sigma_{\tilde{\lambda}}$, where $\tilde{\lambda}=\lambda-\epsilon>\lambda_{1}$.

Proof. For $\delta>0$ (which will be determined later), let $D \subset \Sigma_{\lambda}$ be a closed set such that $\left|\Sigma_{\lambda} \backslash D\right| \leq \delta$. Let $\epsilon>0$ be small enough such that

$$
\left\{\begin{array}{l}
\text { (i) } \tilde{\lambda}:=\lambda-\epsilon>\lambda_{1} \\
\text { (ii) }\left|\Sigma_{\tilde{\lambda}} \backslash \Sigma_{\lambda}\right| \leq \delta \\
\text { (iii) } u\left(x^{\tilde{\lambda}}\right)-u(x)>0 \text { for any } x \in D .
\end{array}\right.
$$

To achieve (iii), we should notice that $u(x)<u\left(x^{\lambda}\right)$ for any $x \in D ; D$ is closed and $u$ is continuous. Set

$$
\Sigma_{\tilde{\lambda}}^{-}=\left\{x \in \Sigma_{\tilde{\lambda}}: u(x)>u\left(x^{\tilde{\lambda}}\right)\right\} .
$$

Then we see that (3.4) still holds with $\Sigma_{\lambda}^{-}$replaced by $\Sigma_{\tilde{\lambda}}^{-}$. If $p \leq 1$, we choose $\delta$ to satisfy $(2 \delta)^{1-p+\frac{\alpha}{n}} \leq \frac{\alpha}{2 A n \omega_{n}^{1-\frac{\alpha}{n}}}$. If $p>1$, since

$$
\left|\Sigma_{\tilde{\lambda}}^{-}\right| \leq\left|\Sigma_{\tilde{\lambda}}^{-} \backslash \Sigma_{\lambda}\right|+\left|\Sigma_{\lambda} \backslash D\right| \leq 2 \delta
$$


we choose $\delta$ small enough such that $C\left\|u^{\frac{n}{\alpha}(p-1)}\right\|_{L^{\frac{n}{\alpha}(p-1)}\left(\Sigma_{\tilde{\lambda}}^{-}\right)}^{p-1} \leq \frac{1}{2}$, where $C$ is the same constant as in (3.6). Then by the same arguments used to show (3.5), we have $\Sigma_{\tilde{\lambda}}^{-}=\emptyset$.

Lemma 3.4. Suppose that as $\lambda=\lambda_{1}$ the first case of (3.1) occurs; that is, $\Sigma_{\lambda_{1}}^{\prime}$ is internally tangent to $\partial \Omega$ at $\tilde{z} \notin T_{\lambda_{1}}$. If $u(x) \leq u\left(x^{\lambda_{1}}\right)$ for any $x \in \Sigma_{\lambda_{1}}$, then $\Sigma_{\lambda_{1}} \cup\left(\Omega \cap T_{\lambda_{1}}\right) \cup \Sigma_{\lambda_{1}}^{\prime}=\Omega$.

Proof. Suppose by contradiction that $\Sigma_{\lambda_{1}} \cup\left(\Omega \cap T_{\lambda_{1}}\right) \cup \Sigma_{\lambda_{1}}^{\prime} \neq \Omega$. This is equivalent to $\Omega_{\lambda_{1}} \neq \emptyset$. Therefore we see that (3.7) still holds with $x$ replaced by $\tilde{z}, x^{\lambda}$ by $\tilde{z}^{\lambda_{1}}$ and $\Omega_{\lambda}$ by $\Omega_{\lambda_{1}}$ respectively. But $\tilde{z}, \tilde{z}^{\lambda_{1}} \in \partial \Omega$ and on the boundary $u$ is constant. This is a contradiction.

Lemma 3.5. Suppose that as $\lambda=\lambda_{1}$ the second case of (3.1) occurs; that is, $T_{\lambda_{1}}$ is orthogonal to $\partial \Omega$ at some point $\hat{z}$. If $u(x) \leq u\left(x^{\lambda_{1}}\right)$ for any $x \in \Sigma_{\lambda_{1}}$, then $\Sigma_{\lambda_{1}} \cup\left(\Omega \cap T_{\lambda_{1}}\right) \cup \Sigma_{\lambda_{1}}^{\prime}=\Omega$.

Proof. From the assumptions, it is easy to see that

$$
\partial_{x_{1}} u(\hat{z})=0 \text {. }
$$

If $\Sigma_{\lambda_{1}} \cup\left(\Omega \cap T_{\lambda_{1}}\right) \cup \Sigma_{\lambda_{1}}^{\prime} \neq \Omega$ or $\Omega_{\lambda_{1}} \neq \emptyset$, then there exists a ball $B \subset \Omega_{\lambda_{1}}$. Let $\left\{x^{m}\right\}_{m=1}^{\infty} \subset \partial \Sigma_{\lambda_{1}} \backslash T_{\lambda_{1}}$ such that $x^{m} \rightarrow \hat{z}$. It follows that $\left(x^{m}\right)^{\lambda_{1}} \rightarrow \hat{z}$. Since $B$ lies on the left of $\hat{z}$, without loss of generality, we assume that $B$ lies on the left of $\left\{\left(x^{m}\right)^{\lambda_{1}}\right\}_{m=1}^{\infty}$. More precisely, we assume that there exists $\delta>0$ such that $\left(x^{m}\right)_{1}^{\lambda_{1}}-y_{1} \geq \delta$ for any $\left(x^{m}\right)^{\lambda_{1}}=\left(\left(x^{m}\right)_{1}^{\lambda_{1}}, \ldots,\left(x^{m}\right)_{n}^{\lambda_{1}}\right)$ and $y=\left(y_{1}, \ldots, y_{n}\right) \in B$.

For any $y \in B$, let $\bar{x}^{m}$ be on the segment from $\left(x^{m}\right)^{\lambda_{1}}$ to $x^{m}$ such that

$$
\frac{1}{\left|\left(x^{m}\right)^{\lambda_{1}}-y\right|^{n-\alpha}}-\frac{1}{\left|x^{m}-y\right|^{n-\alpha}}=-(n-\alpha) \frac{\left(\bar{x}^{m}-y\right)\left(\left(x^{m}\right)^{\lambda_{1}}-x^{m}\right)}{\left|\bar{x}^{m}-y\right|^{n-\alpha+2}} .
$$

Since $\left(x^{m}\right)_{1}^{\lambda_{1}} \leq \bar{x}_{1}^{m} \leq x_{1}^{m}$, we have

$\left(\bar{x}^{m}-y\right)\left(x^{m}-\left(x^{m}\right)^{\lambda_{1}}\right)=\left(\bar{x}_{1}^{m}-y_{1}\right)\left(x_{1}^{m}-\left(x^{m}\right)_{1}^{\lambda_{1}}\right) \geq \delta\left(x_{1}^{m}-\left(x^{m}\right)_{1}^{\lambda_{1}}\right)=\delta\left|x^{m}-\left(x^{m}\right)^{\lambda_{1}}\right|$.

It is easy to see that the first inequality of (3.7) still holds with $x$ replaced by $x^{m}, x^{\lambda}$ by $\left(x^{m}\right)^{\lambda_{1}}$ and $\Omega_{\lambda}$ by $\Omega_{\lambda_{1}}$ respectively for any $m$. Combining this with the above two inequalities, we have

$$
\begin{gathered}
u\left(\left(x^{m}\right)^{\lambda_{1}}\right)-u\left(x^{m}\right) \geq A \int_{\Omega_{\lambda_{1}}} u^{p}(y)\left(\frac{1}{\left|\left(x^{m}\right)^{\lambda_{1}}-y\right|^{n-\alpha}}-\frac{1}{\left|x^{m}-y\right|^{n-\alpha}}\right) d y \\
\geq A(n-\alpha) \delta \int_{B} u^{p}(y) \frac{\left|\left(x^{m}\right)^{\lambda_{1}}-x^{m}\right|}{\left|\bar{x}^{m}-y\right|^{n-\alpha+2}} d y
\end{gathered}
$$

that is,

This contradicts (3.8).

$$
\liminf _{m \rightarrow \infty} \frac{u\left(\left(x^{m}\right)^{\lambda_{1}}\right)-u\left(x^{m}\right)}{\left|\left(x^{m}\right)^{\lambda_{1}}-x^{m}\right|}>0 .
$$

Proof of (3.2). From Lemma 3.1, we see that

$$
\left\{\lambda: u(x) \leq u\left(x^{\lambda}\right) \text { for any } x \in \Sigma_{\lambda} \text { and } \Sigma_{\lambda}^{\prime} \subset \Omega\right\} \neq \emptyset .
$$

Let

$$
\hat{\lambda}=\inf \left\{\lambda: u(x) \leq u\left(x^{\lambda}\right) \text { for any } x \in \Sigma_{\lambda} \text { and } \Sigma_{\lambda}^{\prime} \subset \Omega\right\}
$$


If $\lambda_{1}<\hat{\lambda}$, then from Lemma 3.2, we have $u(x)<u\left(x^{\hat{\lambda}}\right)$ for any $x \in \Sigma_{\hat{\lambda}}$; and then by Lemma 3.3, we have a contradiction since $\hat{\lambda}$ can be decreased a little bit. Therefore $\lambda_{1}=\hat{\lambda}$ (by Lemmas 3.4 and $3.5, \hat{\lambda} \nless \lambda_{1}$ ).

(3.2) (i) is a direct consequence of Lemma 3.2. By Lemmas 3.4 and 3.5, we have (3.2) (ii). Choosing $-x_{1}$ as the $x_{1}$-axis, we obtain (3.2) (iii).

\section{REFERENCES}

1. W.X. Chen, C.M. Li and B. Ou, Classification of solutions for an integral equation, Comm. Pure and Appl. Math. LIX (2006), 330-343. MR2200258 (2006m:45007a)

2. B. Gidas, W. Ni and L. Nirenberg, Symmetry and related properties via the maximum principle, Comm. Math. Phys. 68 (1979), 209-243. MR544879 (80h:35043)

3. J. Serrin, A symmetry problem in potential theory, Arch. Rat. Mech. Anal. 43 (1971), 304-318. MR0333220 (48:11545)

4. G. Ströhmer, About the linear stability of the spherically symmetric solution for the equations of a barotropic viscous fluid under the influence of self-gravitation, J. Math. Fluid Mech. 8 (2006), 36-63. MR2205150 (2006i:76089)

5. G. Ströhmer and W. Zajaczkowski, On the existence and properties of the rotationally symmetric equilibrium states of compressible barotropic self-gravitating fluids, Indiana Univ. Math. J. 46 (1997), 1181-1220. MR.1631576 (99g:76124)

College of Science, Xi'an Jiaotong University, Xi'an 710049, People's Republic of CHINA

E-mail address: lidsh@mail.xjtu.edu.cn

Department of Mathematics, The University of Iowa, Iowa City, Iowa 52242-1419

E-mail address: strohmer@math.uiowa.edu

Department of Mathematics, The University of Iowa, Iowa City, Iowa 52242-1419

E-mail address: lwang@math.uiowa.edu 